\title{
METODOLOGÍAS EN LA ENSEÑANZA DE LA DERIVADA: URACCAN-NUEVA GUINEA
}

\author{
William Oswaldo Flores López ${ }^{[1]}$ \\ María Jesús Salinas Portugal ${ }^{[2]}$
}

\section{Resumen}

Esta investigación describe la metodología que utilizan profesores de la URACCAN -Nueva Guinea, en la enseñanza-aprendizaje de la Derivada a estudiantes de Administración de Empresas, y cómo esta metodología educativa influye en el rendimiento de la asignatura "Matemática Financiera". Fue un estudio de caso en el cual participaron dos profesores de matemática; los datos fueron obtenidos a través de un cuestionario y entrevista abierta; además, una encuesta que se le suministró al alumnado en las que se les solicitó información relacionada a los procesos metodológicos en el aula. El análisis se desarrolló en función de las redes sistémicas y los resultados muestran que existen diferencias significativas a la hora de abordar el contenido, los resultados comparativos favorecen a los estudiantes a quienes se les facilitó clases en la enseña de la Derivada con pertinencia al contexto social y en cuanto al profesor que aborda la Derivada desde el enfoque tradicional, se evidencian calificaciones bajas por parte del estudiantado.

Palabras Clave: Metodología, Administración de Empresas, contexto social, rendimiento, enseñanza-aprendizaje de la Derivada, Matemática Financiera.

\section{Summary}

This research describes the methodology that is been used by teachers of URACCANNueva Guinea, in the teaching and learning of the derivatives with students of Business Administration, and how this educational methodology influences the performance of "Financial Mathematics" as a subject. In this case-study two mathematics teachers participated; the data were obtained through a questionnaire and open interviews, as well as a survey that was provided to the students in which they requested information related to the methodological processes in the classroom. The analysis was developed based on the systemic networks and the results demonstrated significant differences in addressing the content. The comparative results favor students, due to the fact that they were provided with training classes related to the derivative with

[1] Máster en Investigación en Didácticas de las Ciencias Experimentales y Matemática. Oficial de Programa de la Dirección de Cooperación Externa de la URACCAN. william.flores@uraccan.edu.ni

[2] Doctora en Investigación en Didácticas de las Matemáticas, Tutora de esta Investigación, Profesora Titular de la Escuela Universitaria de la Universidad de Santiago de Compostela, España. mjesus.salinas@usc.es 
pertinence to the social context; and as for the teacher who teaches the derivative from a traditional perspective, it was evident the low grades of the students.

Keywords: Methodology, Business Administration, social context, performance, teaching and learning of the derivative, Financial Mathematics.

\section{Introducción}

Esta investigación analiza si una enseñanza basada en las aplicaciones de la Derivada en el contexto social de la carrera de Administración de Empresas influye positivamente en el rendimiento académico de la asignatura de Matemática Financiera.

La perspectiva es averiguar en estudiantes de Administración de Empresas si al introducir el concepto de Derivada en la asignatura de Matemática Aplicada I, utilizando una metodología basada en las aplicaciones de pertinencia del contexto social de la carrera, mejoraría positivamente el rendimiento de los estudiantes en la asignatura de Matemática Financiera.

Según Artigue (1995), los estudiantes pueden aprender a realizar de forma mecánica cálculos de Derivadas y resolver algunos problemas, se encuentran grandes dificultades para alcanzar una verdadera compresión de los conceptos involucrados y un desarrollo adecuado de métodos de pensamiento que son el centro de este campo de la matemática. Un fenómeno educativo de la matemática es el predominio de métodos algebraicos y algorítmicos.

Hoy en día se ha avanzado en la compresión de la noción de Derivada en los estudiantes de Administración de Empresas. En el aprendizaje del cálculo y en particular en la conceptualización de la Derivada, comprende uno de los desafíos de la educación actual, ya que los educandos son capaces de derivar una función, no reconocen ciertas aplicaciones según el contexto, esto provoca que una gran cantidad de estudiantes no logren dar sentido y significado a los conceptos básicos del cálculo diferencial, en este caso es necesario que estos contenidos se desarrollen según la carrera que estudian los estudiantes.

\section{Revisión Literatura}

\section{Métodos en la enseñanza del concepto de la Derivada}

Ante la constatación de la tendencia en la enseñanza de dedicar una gran parte de las actividades al aprendizaje de reglas de cálculo, sin basarse en la compresión de los conceptos, Azcárate y Cols (1997), resaltan la importancia del uso de representaciones diversas, como puede ser la gráfica, la numérica y algebraica, de manera de relacionarlas y pasar de una a otra a fin de alcanzar representaciones mentales ricas que reflejen 
muchos aspectos relacionados con el concepto. En general, las tareas de conversión entre diferentes sistemas de representación son minimizadas en la enseñanza y eso produce limitaciones en la compresión. Duval (1998) expresa que el uso de distintas representaciones es esencial en el desarrollo del pensamiento y en la producción del conocimiento. Distintos autores apoyan esta idea y manifiestan que llegar a comprender un concepto matemático implica realizar procesos de conversión entre diferentes registros de representación, manifestados por la posibilidad de movilización y de articulación entre los mismo (Rico, 2000, D’Amore, 2002).

Durante los últimos años se ha desarrollado en México una línea de investigación conocida como socio epistemología, la cual estudia los fenómenos de producción y difusión del conocimiento desde una perspectiva múltiple (Cantoral \& Farfán, 2003). Dicho enfoque incorpora el estudio de las interacciones entre la epistemología del conocimiento, la dimensión sociocultural, los procesos cognitivos asociados y los mecanismos de institucionalización por vía de la enseñanza. La perspectiva múltiple también subraya el papel que fungen el pensamiento y el lenguaje variacional para estudiar la Derivada, de ahí que se centre en las prácticas sociales que dan vida a las matemáticas de la variación como una cuantificación del cambio (Cantoral y Farfán, 1998). Los trabajos que recurren a esta línea de investigación defienden la idea de que hasta que no se vea la noción de Derivada como una organización de las variaciones sucesivas no será comprendida (Cantoral y Farfán, 1998).

\section{Aplicaciones de la Derivada en la carrera de Administración de Empresas}

La Derivada aplicada al contexto social de la carrera de Administración de Empresas es una herramienta muy útil puesto que por su naturaleza permite realizar cálculos marginales, es decir hallar la razón de cambio cuando se agrega una unidad adicional al total, sea cual sea la cantidad económica que se esté considerando: costo, ingreso, beneficio o producción. Tal línea de pensamiento fue posible desde la economía neoclásica, primero con Carnot, y luego con León Walras, Stanley Jevons y Alfred Marshall; por ello se conoce a esta innovación analítica como la revolución marginalista. De hecho las funciones de costo, ingreso, beneficio o producción marginal son las Derivadas de las funciones de costo, ingreso, beneficio, producción total.

En ese orden de ideas, el procedimiento se reitera en el contexto de las funciones multivariadas. Mediante las Derivadas parciales, es decir estimar las razones de cambio de una variable independiente de una $\mathrm{f}(\mathrm{x}, \mathrm{y})$ son las Derivadas parciales respecto a $\mathrm{x}$ o y, manteniendo la(s) otra(s) fija(s). En consecuencia se pueden aplicar las técnicas especiales como Derivadas direccionales, gradientes, diferenciales.

Se requiere con frecuencia estimar los niveles donde una función cualquiera se maximiza (minimiza), sea cual sea el número involucrado de variables independientes. También para la búsqueda de la optimización sujeta a restricciones se trata con 
derivación de las funciones mediante los métodos de los multiplicadores de Lagrange o las condiciones de Kühn-Tucker.

\section{Materiales y métodos}

El enfoque es de tipo cualitativo, mientras que su naturaleza se define como descriptiva, exploratoria e interpretativa. El tipo de investigación que se diseñará para tal fin es el estudio de casos ya que el objetivo general de nuestro estudio es: "estudiar dos metodologías utilizadas en la enseñanza-aprendizaje de la Derivada con los estudiantes de Administración de Empresas y su influencia en el rendimiento de la asignatura de Matemática Financiera que implica el conocimiento del concepto de la Derivada"

Se estudió el rendimiento de los estudiantes de la carrera de Administración de Empresas de la URACCAN-Nueva Guinea, en la asignatura de Matemática Financiera al finalizar el curso 2010-2011. Estos estudiantes habían cursado, anteriormente, la asignatura de Matemática Aplicada I que incluye, por primera vez, el estudio de la Derivada. A una parte de los estudiantes le impartió clases el profesor A, en cuya metodología se contemplan las aplicaciones de la Derivada en el contexto social de la carrera y, a la otra parte le impartió clases el profesor B que no utiliza las aplicaciones de la Derivada en el contexto social de la carrera citada anteriormente.

Llamaremos grupo A al grupo formado por los estudiantes a los que les impartió clases en la asignatura de Matemática Aplicada I el profesor A, y grupo B al grupo formado por los estudiantes a los que les impartió clases el profesor B en dicha asignatura. Cada grupo estaba formado por 16 estudiantes.

Técnicas e instrumentos: Hemos construido un cuestionario y se realizó una entrevista, ambos destinados a cada uno de los profesores objeto de estudio. También se realizó una encuesta a los estudiantes para completar la información anterior y poder triangular los resultados.

\section{Resultados y discusión}

Se obtuvo el rendimiento de los estudiantes de la carrera de Administración de Empresas en la asignatura de Matemática Financiera a través del resultado de sus calificaciones. Se dividieron dos grupos: Grupo A los estudiantes que habían cursado la asignatura Matemática Aplicada I con el profesor A; grupo B los estudiantes que habían cursado dicha asignatura con el profesor B. Recordamos que en la metodología del profesor A se contemplan las aplicaciones de la Derivada en el contexto social de la carrera, mientras que el profesor B no utiliza dicha aplicación. 
La media de las calificaciones de los estudiantes podemos verla en el cuadro No. 1:

Cuadro No. 1. Rendimiento en Matemática Financiera

\begin{tabular}{|l|r|r|r|}
\hline $\begin{array}{c}\text { Grupos de } \\
\text { Matemática } \\
\text { Aplicada I }\end{array}$ & No alumnos & $\begin{array}{c}\text { Nota media } \\
\text { en M. Financiera }\end{array}$ & \multicolumn{2}{|c|}{$\begin{array}{c}\text { Desviación } \\
\text { típica }\end{array}$} \\
\hline Grupo A & 16 & 66 & 10,8 \\
\hline Grupo B & 16 & 54 & 8,2 \\
\hline
\end{tabular}

Podemos ver que la nota media en la asignatura Matemática Financiera de los estudiantes del grupo A es mayor que la media de la nota del grupo B. Para ver si esta diferencia es significativa se aplicó un ANOVA, cuyos resultados presentamos en el cuadro No. 2:

Cuadro No. 2. Análisis de la varianza: Rendimiento en Matemática Financiera

\begin{tabular}{|l|r|r|r|r|r|}
\hline \multicolumn{7}{|c|}{ ANOVA } \\
\hline & $\begin{array}{c}\text { Suma de } \\
\text { cuadrados }\end{array}$ & gl & Media cuadrática & F & Sig. \\
\hline Inter-grupos & 1262,531 & 1 & 1262,531 & 12,899 &, 001 \\
\hline Intra-grupos & 2936,438 & 30 & 97,881 & & \\
\hline Total & 4198,969 & 31 & & & \\
\hline
\end{tabular}

Como puede observarse en el análisis de la varianza (ANOVA), el valor de F es significativo para $\mathrm{a}=0,05$ ya que $\mathrm{p}=0,001$ menor que $\mathrm{a}$. Por lo tanto, podemos afirmar que el grupo A tiene mejores resultados en la asignatura de Matemática Financiera respecto al grupo B. Podíamos pensar que las causas de estas diferencias estuvieran en la metodología empleada por el profesor A como más motivadora que la que utiliza el profesor B; sin embargo, para Johnson (1985) la motivación para aprender consta de muchos elementos entre los que se incluyen: Planificación, concentración en la meta, consciencia metacognoscitiva de lo que se pretende aprender y como se pretende aprenderlo, búsqueda activa de una nueva información, percepciones claras de la retroalimentación, elogio y satisfacción por el logro y ninguna ansiedad al fracaso o temor al fracaso. Lorenzo (2002, p. 157) añade: "ya que el nivel de motivación está relacionado con el grado de compresión de las y los estudiantes, este manejo de elementos motivacionales no depende solo de la elección de la estrategia que utilizará la o él docente, sino también con la forma en que éste es presentado a las y los estudiantes".

Respecto a la importancia de la Derivada en los contenidos de curso de Matemática Aplicada, para la carrera de Administración de Empresas ambos profesores consideran importante su estudio, pero con distintos enfoques como se puede apreciar en la red sistémica correspondiente. 


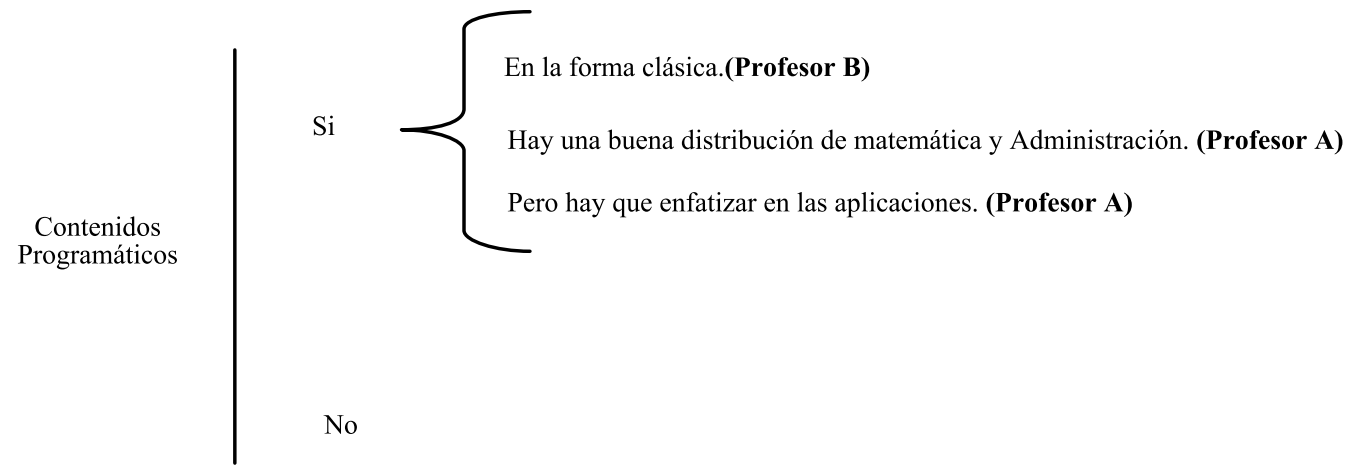

\section{Red Sistémica 1}

\section{Las repuestas de los dos profesores de Matemáticas}

Profesor B: El concepto de Derivada y su interpretación geométrica permite entender técnicas para deducir cuando una función crece o decrece. Así mismo, tiene importancia porque la Derivada permite obtener información sobre comportamientos de una o varias variables respecto a otras. También se puede interpretar como la Derivada respecto al tiempo. Muchos de los problemas que se presentan en la economía se pueden modelar desde un punto de vista matemático. En problemas como la optimización o aquellos donde el comportamiento de ciertos elementos depende de la variación de otros es muy útil la teoría de las Derivadas.

Profesor A: La Derivada es el pilar fundamental de los contenidos para las carreras de Administración de Empresas, puesto que tiene muchas aplicaciones y son útiles para resolver muchos problemas de economía, son de gran utilidad ya que los estudiantes aprenden bien su aplicación, porque se desarrollan en el contexto-social de la carrera desarrollando importantes conceptos de economía como puede ser la razón de cambio, marginalidad, optimización, el estudio, análisis e interpretación de tales conceptos. La Derivada es sumamente importante para entender las tasas marginales que se utilizan en las aplicaciones a la economía.

Los dos profesores consideran importante el estudio de la Derivada como una herramienta a la hora de resolver problemas matemáticos y económicos, pero se aborda desde distintos enfoques: El profesor B utiliza un conocimiento de la Derivada como un objeto matemático para el análisis de situaciones económicas representadas en modelos matemáticos. El profesor A resalta la importancia de la Derivada en el currículum de las carreras de Administración de Empresas como una herramienta para el análisis e interpretación de los modelos, tanto económicos como matemáticos. 
Estructura que usa el profesor para desarrollar el tema de las aplicaciones de la Derivada

Podemos destacar que el profesor A, desarrolla el tema de la Derivada, con el análisis marginal, la razón de cambio relativa y porcentual y la función de consumo; relaciona la Derivada con gráfica de funciones y optimización; asimismo, los problemas de utilidad, costos, demandas de consumidores, eficiencia del trabajador, impuesto, máximos y mínimos. Por otra parte, el profesor B inicia el tema de la Derivada con la interpretación geométrica, utiliza la Derivada para determinar intervalos de crecimiento, puntos extremos, intervalos donde la gráfica de una función cóncava hacia arriba y hacia abajo.

\section{En cuanto a las estrategias metodológicas}

Profesor A. Plantea problemas concretos para que los estudiantes participen en su resolución. Esto permite analizar el problema identificando fenómenos, variables, constantes, interdependencia de los elementos involucrados y abra pautas para un modelo representativo algebraico que se adapte a la situación planteada.

Profesor B. La estrategia que utiliza es dar ejemplos clásicos a través de definiciones rigurosas, diferentes aplicaciones físicas. También utiliza trabajos en equipo que permiten que los estudiantes reflexionen sobre las distintas formas de abordar los problemas.

Utilizando los resultados de la encuesta a los estudiantes, estos afirman que ambos profesores recurren a las experiencias previas del estudiantado, así como a estrategias de búsqueda de información previa a las clases por lo que desarrollan clases participativas. Estos profesores utilizan las tecnologías de la información y comunicación. Por otra parte, el uso de la geometría como mecanismo de enseñanza de la Derivada es una herramienta esencial en este proceso de enseñanza.

\section{Procedimiento de evaluación:}

Los profesores A y B toman en cuenta la evaluación diagnóstica puntual al inicio, durante y al finalizar su clases, como un referente para lograr objetivos propuesto; registran los pre saberes de los estudiantes al hacer preguntas claves o generadoras de conceptos e ideas varias sobre un determinado tema. Asímismo, los profesores de matemática toman en cuenta los resultados de la evaluación diagnóstica; presentan algunas alternativas o medidas para mejorar el aprendizaje del estudiante, facilitando la comprensión del tema a desarrollar. También tienen en cuenta la participación del estudiantado en el transcurso de los diferentes momentos del aprendizaje. 
Las diferencias de los procesos educativos entre discente y docente del respecto al concepto de Derivada y la posible implementación de elementos innovadores.

Las categorías que surgen por parte de los profesores $\mathrm{A}$ y $\mathrm{B}$, cuando se les pregunta acerca de la introducción del concepto de la Derivada y de la interpretación que hacen sobre de ella para llegar propiamente al concepto, son las que sugieren los programas oficiales: Razón de cambio e interpretación geométrica. Por otra parte, ningún profesor habló sobre propuestas alternativas o no tradicionales para la enseñanza de la Derivada en el campo que nos ocupa por ejemplo a través de la utilidad marginal (Arya y Lardner, 1987) o del impuesto marginal (Wonnacott, 1983).

La anterior situación podemos verla en la red sistémica No. 2:

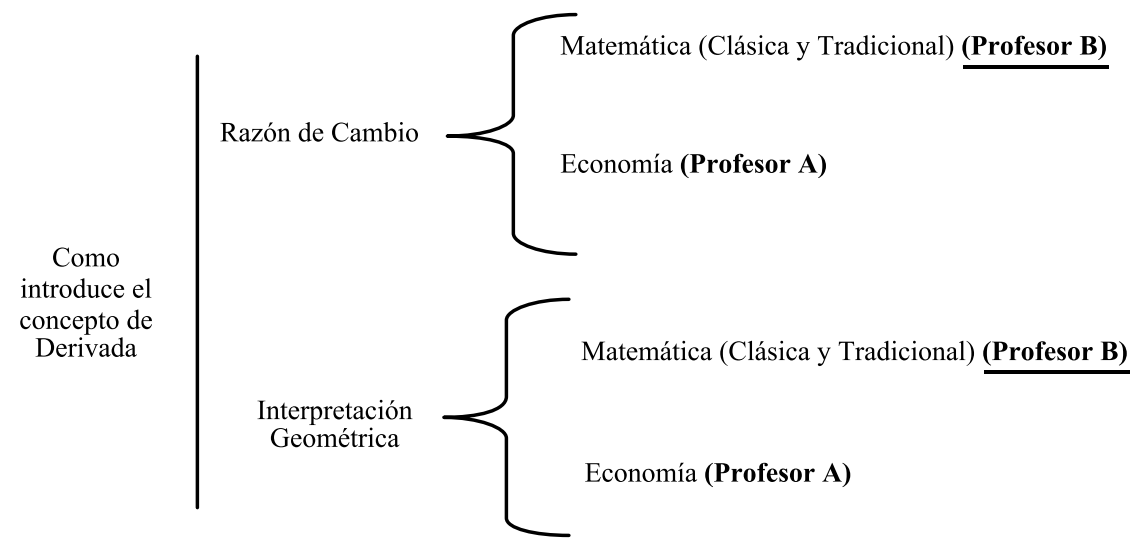

\section{Red Sistémica 2}

Razón de Cambio: El profesor $\mathbf{B}$ afirma que introduce la razón de cambio y pendiente con un enfoque puramente matemático o más bien físico, mientras que el Profesor A, dice quese tiene que hacer hincapié en la definición de la Derivada como razón de cambio, pero siempre vinculando este concepto con el análisis marginal.

Interpretación geométrica: El profesor B, opina que prefiere utilizar la interpretación geométrica,considerando la baja calidad de los aprendizajes matemáticos que poseen los estudiantes. Por otra parte, el profesor A, manifiesta que le parece muy importante la interpretación geométrica, unida a la explicación conceptual del término a nivel del contexto-social de la carrera de Administración de Empresas, para entender y visualizar los procesos que suceden. 
Respecto a la segunda parte de la pregunta, que está asociada con el ejemplo para introducir el concepto de Derivada en la carrera de Administración de Empresas, las conclusiones del análisis indican que el profesor B, al enseñar el concepto de Derivada, le da un fuerte peso al contexto matemático en sí, descuidando su relación con la carrera, mientras que el profesor A, contextualiza las situaciones reales del estudiantado.

Podemos ver esta situación en la red sistémica No. 3:

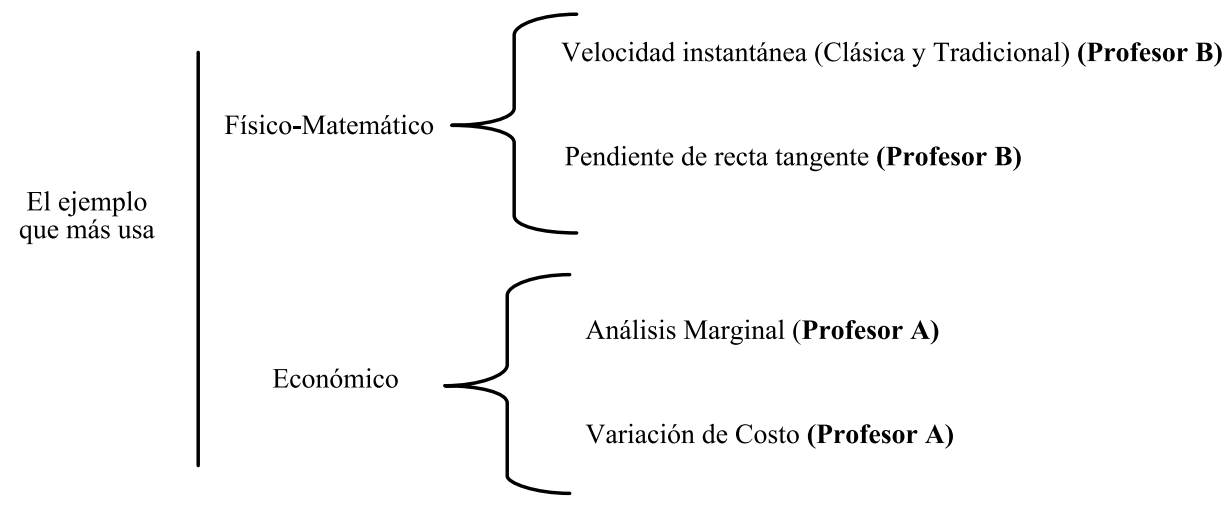

\section{Red Sistémica 3}

Cuando se pide a los profesores participantes que den su opinión acerca de una propuesta de trabajo más próxima a la identificación de situaciones reales que puedan ser modelizadas y resueltas matemáticamente, las respuestas fueron las siguientes:

El profesor A, manifiesta que es factible introducir el concepto utilizando un problema que representa una situación de la vida cotidiana de un Administrador de Empresas, pero sería importante consultar a los profesores del área de Ciencias Administrativas para saber cuál ha sido su experiencia desde esta perspectiva de enseñanza. También propone que es necesario utilizar recursos didácticos.

El profesor B, considera que lo hecho en la actualidad es lo más apropiado en cuanto a estrategia pedagógica se refiere.

\section{Conclusiones}

En cuanto al objetivo principal de esta investigación: Averiguar si una enseñanza basada en las aplicaciones de la Derivada en el contexto-social de la carrera de Administración de Empresas, influye positivamente en el rendimiento académico de la asignatura de Matemática Financiera que implica el conocimiento del concepto de la Derivada. Vemos que los estudiantes, a los que se les introdujo el concepto de Derivada a través de sus aplicaciones en el contexto-social, tuvieron mejor rendimiento 
en la asignatura Matemática Financiera que aquellos a quienes se les introdujo este concepto de forma clásica.

Acerca de la estructura, procedimiento metodológicos y de evaluación que el profesor utiliza para desarrollar el proceso de enseñanza-aprendizaje de la Derivada, resaltan los siguientes aspectos:

El profesor B, sigue utilizando una metodología tradicional para enseñar el concepto de la Derivada basada en aspectos fisicomatemáticos o geométricos, descartando alternativas innovadoras relacionadas con la pertinencia de la carrera de Administración de Empresas; mientras que el profesor A, mantiene una línea de instrucción transformadora del concepto de la Derivada, a través de la relación con las aplicaciones que viven los estudiantes en su carrera.

Se detectan algunas carencias didácticas relacionadas con la pertinencia del contexto, por un lado, la convivencia de un intercambio de experiencias didácticas entre profesores, por otro, algún tipo de formación didáctica y conceptual relacionada con el contenido económico. En cuando el conocimiento del contenido económico es parcial.

\section{Lista de referencias}

Artigue, M. (1995). La enseñanza del principio del cálculo: problemas epistemológicos, cognitivos y didácticos. Grupo Editorial Iberoamérica. Bogotá, Colombia.

Azcárate, C. \& Cols. (1997). Cálculo diferencial e integral. España: Síntesis.

Cantoral, R. \& Farfán, R. (1998). Pensamiento y lenguaje variacional en la introducción al análisis. Épsilon 42, 353-369.

Cantoral, R. \& Mirón, H. (200o). Sobre el estatus de la noción de Derivada: de la epistemología de Joseph Louis LaGrange al diseño de una situación didáctica. Revista latinoamericana de Investigación en Matemática Educativa. 3(3), pp. 265-292.

Cantoral, R. \& Farfán, R. (2003). Mathematics education: a vision of its evolution. Educational Studies in Mathematics 53 (3), 255-270.

D’Amore, B. (2002). La complejidad de la noética en matemática como causa de la falta de devolución. Revista TED de la Universidad Pedagógica de Bogotá. Colombia. 
Duval, R. (1998). Registros de representación semiótica y funcionamiento cognitivo del Pensamiento. En HITT, F. (Ed.). Investigaciones en Matemática Educativa II. pp. 173-201. Grupo Editorial Iberoamérica: México. Traducción de: Registres de représentationsémiotique et functionnementcognitif de la pensée. Annales de Didactique et de Sciences Cognitives. Vol. 5 (1993).

Rico, L. (2000). Sobre las nociones de representación y comprensión en la investigación en Educación Matemática. IV Simposio SEIEM. Huelva. España. 